\title{
The Latest on a Challenging Diagnostic Entity
}

doi: 10.1017/S1355617708080648

\begin{abstract}
Mild Traumatic Brian Injury and Postconcussion Syndrome: The New Evidence Base for
Diagnosis and Treatment, by Michael A. McCrea. 2007. New York: Oxford University Press. 224 pp., \$39.95 (PB).
\end{abstract}

Reviewed by Ty S. Callahan, Ph.D., ABPP, Neuropsychological Consulting, Omaha, NE, USA.

Few topics in neuropsychology, or healthcare for that matter, have been as controversial for as long a time as mild traumatic brain injury (MTBI) and postconcussive syndrome (PCS). The controversy spills from healthcare into the medico-legal arena for obvious reasons, but is far from being only an issue of interest in forensic circles. Patients with MTBI who report symptoms of PCS are among the most common customers in clinics around the world. The controversy persists, in large part, because of the challenges associated with objectively capturing, describing and understanding the issue. Dr. McCrae's book speaks directly to these challenges by reviewing the dramatic increase in research on MTBI and PCS over the last ten or so years.

Mild Traumatic Brian Injury and Postconcussion Syndrome: The New Evidence Base for Diagnosis and Treatment is the second volume in a relatively new series resulting from a joint effort of the American Academy of Clinical Neuropsychology (AACN) and Oxford University Press. The series sets out to present timely, focused, applied information from selected annual AACN Workshops. MTBI and PCS have been among the most elusive and contested diagnostic entities for clinicians. As Dr. McCrea points out, the clinical challenge and prevalence of MTBI juxtaposed with significant recent advances in the relevant basic and clinical sciences makes this text as timely and as welcome as any in recent years.

The text aims to serve this end by surveying with enough breadth to assure no piece of the "big picture" is overlooked, while providing sufficient depth of review so as to instill in the reader a comfortable level of confidence about which conclusions and recommendations for action are valid. Twenty-one compact, but readable chapters are organized into four Parts covering 1) the epidemiological and definitional context of MTBI, 2) advances in pathophysiology and neuroimaging of MTBI, 3) integration of "old" and "new" empirical information on the natural course of MTBI, and 4) an up-to-date informed review of PCS with concrete, empirically based recommendations for intervention.

Information provided in Part One, The TBI Landscape, on the incidence and societal impact of traumatic brain injury is up-to-date, but the end result is not particularly course changing. Few have previously argued against the notion of traumatic brain injury, including MTBI, being both frequent and costly. The most significant contribution of this section involves the theoretical introduction to sport concussion. The section is significant not because it holds sport concussion research up as neuropsychology's relatively unique and interesting contribution to the wide world of sports but, instead, because Dr. McCrea carefully explains the important theoretical contribution that the study of sport concussion has made towards better understanding MTBI in general.

Part Two, Basic and Clinical Science of MTBI, covers biomechanics, trauma-related neurometabolic dysfunction, and neuroimaging of MTBI. Chapters on the biomechanics of, and subsequent neurometabolic cascade following, MTBI are likely among the most informative chapters of the book for clinicians most distant from academic medical settings. These very readable summaries of technical information include attempts to make direct connections with applied clinical practice. Clinicians will likely appreciate the identified correlation between current understanding of the temporal aspects of the neurometabolic cascade and the observed clinical course of MTBI. While discussion of the biomechanics of MTBI is no less informative, direct application of this information is slightly more challenging. Dr. McCrea's recommendation to consider evidence of the minimal biomechanical threshold for injury certainly follows directly from the science reviewed, but leaves the reader wanting slightly more guidance with respect to how this might be accomplished. Those less fluent in physics will have difficulty determining the likelihood that a particular fall on an icy sidewalk or a blow to the head from falling lumber could generate the $80 \mathrm{~g}$ to $100 \mathrm{~g}$ acceleration necessary for causing a MTBI. Setting aside this challenge of application to clinical practice, the information offered on the biomechanics of brain injury and concussion is extremely timely, clear, and thorough. The chapter on neuroimaging very competently reviews the latest literature on traditional and newer imaging techniques, but the gist of the chapter will perhaps be more exciting to researchers than clinicians, because of the number of unanswered questions with respect to MTBI outcome rather than guidelines for the practitioner.

Part Three, The Natural History of MTBI, will perhaps be the most resonant for the clinician as it is an excellent and timely review of a wide ranging literature on the course of MTBI and PCS, and of reported symptoms, objective cognitive performance, and functional outcome. Part Four, Implications for Rethinking Postconcussion Syndrome, directly addresses PCS, is clearly the centerpiece of the text, and is directly supported by the previously reviewed information.

Dr. McCrea expertly takes on the historical problems of PCS diagnosis. including usefulness of formal diagnostic schemes, revised estimates of true incidence, and the diverse factors involved in causation and maintenance of PCS. Dr. 
McCrea's discussion of PCS as a uniquely neuropsychological disorder very nicely anticipates and dovetails with a recent proposal for designation of Cogniform Disorder and Cogniform Condition diagnoses (Delis \& Wetter, 2007). His treatment recommendations for PCS are straightforward and practical, but perhaps more powerfully asserted given Dr. McCrea's comprehensive and cohesive discussion of the empirical literature upon which the recommendations are based.

Mild Traumatic Brian Injury and Postconcussion Syndrome: The New Evidence Base for Diagnosis and Treatment is a jewel. It is rare in its accomplishment to so succinctly cover such an impressive amount of research.

\section{RECENT AND RELEVANT}

doi: $10.1017 /$ S1355617708080661

History of Psychiatry and Medical Psychology. Edwin R. Wallace, IV and John Gach (Eds.). 2008. Secaucus, NJ: Springer, 862 pp., \$89.95 (HB).

Bayesian Brain: Probabilistic Approaches to Neural Coding. Kenji Doya, Shin Ishii, Alexandre Pouget, and Rajesh P. N. Rao, (Eds.). 2007. Cambridge, MA: The MIT Press, 326 pp., \$54.00 (HB).

Treating Tourette Syndrome and Tic Disorders: A Guide for Practitioners. 2007. Douglas W. Woods, John C. Piacentini, and John T. Walkup (Eds.). New York: The Guilford Press, 287 pp., \$38.00 (HB).

\section{A Clinician's Handbook of Child and Adolescent} Psychiatry. Christopher Gillberg, Richard Harrington, and Hans-Christoph Steinhausen (Eds.). 2006. New York: Cambridge University Press, 751 pp., $\$ 100.00$ (HB).
Because of this, it will appeal to all levels of readers with an interest in MTBI and PCS. Old hands and students alike will find this book a concise and comprehensive reference text on the topic of MTBI and PCS. The added benefit of being able to obtain continuing education credit for books in the Oxford Workshop Series is a nice touch. I wholeheartedly recommend this book to all with an interest or need.

\section{REFERENCE}

Delis, D.C. \& Wetter, S.R. (2007). Cogniform Disorder and Cogniform Condition: Proposed diagnoses for excessive cognitive symptoms. Archives of Clinical Neuropsychology, 22, 589-604.

Handbook of Mathematical Cognition. Jamie I. D. Campbell (Ed.). 2005. New York, NY: Psychology Press, 211 pp., \$125.00 (HB).

Emotion Regulation: Conceptual and Clinical Issues, by Ad Vingerhoets, Ivan Nyklicek, and Johan Denollet. 2008. Secaucus, NJ: Springer, 246 pp., $\$ 89.95$ (HB).

Handbook of Emotion Regulation. James J. Gross (Ed.). 2006. New York, NY: Guilford Publications, 654 pp., \$85.00 (HB).

Emotions and Aggressive Behavior. Georges Steffgen and Mario Gollwitzer (Eds.). 2007. Seattle, WA: Hogrefe and Huber, 229 pp., US\$52.00; CAN\$ 68.00; €44.95 (HB). 